\title{
SOME APPLICATIONS OF GENERALIZED EXPONENTIALS TO PARTIAL DIFFERENTIAL EQUATIONS
}

\section{J. ABRAMOWICH}

\begin{abstract}
Using what may be considered as a natural generalization of the exponential function, some of the formalism of the theory of ordinary linear differential equations is extended to a class of linear partial differential equations among which are some important equations of mathematical physics.

In $\$ 1$ we give the definitions of the generalized exponentials and derive expressions for them. $\$ 2$ is devoted to the study of some of the properties of the exponential in two independent variables. In $\$ 3$ we derive the general solutions of some key partial differential equations using the method of recursion. The last section is devoted to extending the formalism of the method of variation of parameters to a class of linear partial differential equations.
\end{abstract}

Introduction. The exponential function $\exp (x)$ plays a key role in the theory of ordinary linear differential equations as the function invariant under the operation of differentiation. In attempting to generalize this function as a function of more than one independent variable it is convenient to begin with the definition of $\exp (x)$ as the solution of a certain integral equation which leads to an obvious choice for the generalization to more than one independent variable. These functions enable us to extend some important results of the theory of ordinary differential equations to partial differential equations.

1. General exponentials. Let $x, t, a, b$ denote $n$-vectors, $x=\left(x_{1}, x_{2}, \ldots, x_{n}\right)$, etc. Let $J_{a x}(v(t))$ denote the integral

$$
\int_{a_{1}}^{x_{1}} \cdots \int_{a_{n}}^{x_{n}} v\left(t_{1}, \ldots, t_{n}\right) d t_{1} \cdots d t_{n}
$$

and $R(b)$ the closed region $\left[0, b_{1}\right] \times \cdots \times\left[0, b_{n}\right]$ in the Euclidean space $E^{n}$ and $D$ the differential operator $\partial^{n} / \partial x_{1} \ldots \partial x_{n}$. The definition of $\exp \left(\int_{0}^{x} v(t) d t\right)(x, t$-scalar $)$ as the solution of the integral equation $u(x)=1+\int_{0}^{x} v(t) u(t) d t$ leads naturally to

DEFINITION 1. If $v(t)$ is a continuous function in some neighborhood of the origin, then the solution of

$$
u(x)=1+J_{0 x}(v(t) u(t))
$$

shall be denoted by $E_{n}^{*}(J(v(t)))$ and considered as the generalization of $\exp \left(\int_{0}^{x} v(t) d t\right)$ to $n$ independent variables $x_{1}, \ldots, x_{n}$.

If $n=1$ we shall write $E_{1}^{*}(J(t))=\exp \left(\int_{0}^{x} v(t) d t\right)$.

Received by the editors May 17, 1982.

1980 Mathematics Subject Classification. Primary 35G05.

Key words and phrases. Generalized exponentials, linear partial differential equations, variation of parameters, recursion. 
The solution of (1.1) is most easily obtained by noting that (1.1) may be interpreted as a substitution rule and using the method of recursion. After $m$ substitutions, (1.1) is transformed into

$$
u(x)=\sum_{k=0}^{m} I_{k}(x)+J_{0 x}\left(I_{m}(t) v(t) u(t)\right)
$$

where

$$
I_{0}(x)=1, \quad I_{k+1}(x)=J_{0 x}\left(I_{k}(t) v(t)\right), \quad k \geqslant 0 .
$$

It is easily seen that

$$
\left|J_{0 x}\left(I_{k}(t) v(t) u(t)\right)\right| \leqslant M /((k+1) !)^{n}
$$

so that the series $\sum_{k=0}^{\infty} I_{k}(x)$ converges uniformly in any closed neighborhood of the origin in which $v(t)$ is continuous. Hence

$$
E_{n}^{*}\left(J_{0 x}(v(t))\right)=\sum_{k=0}^{\infty} I_{k}(x)
$$

This is the so-called Neumann series for the function $v(x)$. The uniqueness of the solution is evident. Of special interest is the case that $v(x)=\beta=$ constant in which case

$$
E_{n}^{*}\left(J_{0 x}(\beta)\right)=E_{n}\left(\beta x_{1} x_{2} \cdots x_{n}\right)
$$

where

$$
E_{n}(z)=\sum_{k=0}^{\infty}\left(z^{k} /(k !)^{n}\right)
$$

The function $E_{n}\left(x_{1} x_{2} \ldots x_{n}\right)$ is the generalization to $n$ independent variables of the function $\exp (x)$. The exponential property

$$
D E_{n}^{*}\left(J_{0 x}(v(t))\right)=v(x) E_{n}^{*}\left(J_{0 x}(v(t))\right)
$$

is true and

$$
\left.E_{n}\left(J_{0 x}(v(t))\right)\right|_{x_{i}=0}=1, \quad i=1, \ldots, n
$$

Proposition 1.1. The function $E_{n}(z)$ satisfies the ordinary differential equation

$$
\frac{d}{d z}\left(z \frac{d}{d z}\right)^{n-1} E_{n}(z)=E_{n}(z)
$$

This is clear from (1.6).

Proposition 1.2. Let $E_{n}^{(0)}(z)=E_{n}(z)$ and

$$
E_{n}^{(k)}(z)=\frac{d^{k}}{d z^{k}} E_{n}(z), \quad E_{n}^{(-k)}(z)=\int_{0}^{z} E_{n}^{(-k+1)}(x) d x, \quad k \geqslant 1 .
$$

Then the functions $E_{n}^{(k)}(z)$ possess the generating function

$$
E_{n-1}(t) \exp (z / t)=\sum_{k=-\infty}^{\infty} t^{k} E_{n}^{(k)}(z), \quad n \geqslant 2 .
$$

The proof is a straightforward consequence of (1.6). 
The Bessel functions of nonnegative integral order are expressible in terms of the functions $E_{2}^{(k)}(z)$,

$$
J_{k}(z)=(z / 2)^{k} E_{2}^{(k)}\left(-(z / 2)^{2}\right), \quad k \geqslant 0 .
$$

We shall now establish a result of importance in the following.

Proposition 1.3. Let $u(x)$ be continuous for $x \in R(b)$ and satisfy the integral equation

$$
u(x)=f(x, u(x))+J_{0 x}(v(t) u(t)) .
$$

If $v(x)$ and $f(x, u(x))$ are continuous for $x \in R(b)$ then $u(x)$ satisfies the integral equation

$$
u(x)=f(x, u(x))+J_{0 x}\left(v(t) E_{n}^{*}\left(J_{t x}(v(t))\right) f(t, u(t))\right)
$$

for $x \in R(b)$.

Proof. The proof is based on recursion (not to be confused with the idea of successive approximations). Interpreting (1.10) as a substitution rule, we may substitute the r.h.s. of (1.10) for $u(t)$ under the integral sign without having to do the same for $u(x)$ in the first term on the r.h.s. The first such substitution, after a change in the order of integration, transforms $(1.10)$ into

$$
u(x)=f(x, u(x))+J_{0 x}(v(t) f(t, u(t)))+J_{0 x}\left(v(t) K_{1}(x, t) u(t)\right),
$$

where $K_{1}(x, t)=J_{t x}(v(s))$. Repeating the procedure, we obtain

where

$$
u(x)=f(x, u(x))+J_{0 x}\left(v(t)\left(\sum_{k=0}^{m} K_{k}(x, t)\right) f(t, u(t))\right)+R_{m}
$$

$$
R_{m}=J_{0 . x}\left(v(t) K_{m+1}(x, t) u(t)\right)
$$

and

$$
K_{j+1}(x, t)=J_{t x}\left(v(s) K_{j}(x, s)\right), \quad K_{0}(x, t)=1 .
$$

The estimate (1.3) applies to $R_{m}$ which implies the convergence of the series $\sum_{i=0}^{\infty} K_{i}(x, t)$ uniformly in $x$ for $x \in R(b)$. This proves the validity of (1.11).

If $f(x, u(x))$ does not depend on $u(x)$ then (1.11) would give us the solution of (1.10).

2. The case of two independent variables. In this section we shall derive some properties of the function $E_{2}(z)$ and related functions. From here on we shall abandon vector notation so that $x, y, a, b$, etc. shall denote scalars. $D$ shall denote the mixed partial derivative $\partial^{2} / \partial x \partial y$.

Define

$$
E_{2}(i z)=C_{2}(z)+i S_{2}(z), \quad i^{2}=-1 .
$$

The functions $C_{2}(z)$ and $S_{2}(z)$ are related to the "ber" and "bei" functions of the Bessel family and satisfy the relations

$$
\begin{aligned}
D C_{2}(\beta x y) & =-\beta S_{2}(\beta x y), \quad D S_{2}(\beta x y)=\beta C_{2}(\beta x y), \\
D^{2} C_{2}(\beta x y) & =-\beta^{2} C_{2}(\beta x y), \quad D^{2} S_{2}(\beta x y)=-\beta^{2} S_{2}(\beta x y) .
\end{aligned}
$$


These follow directly from the relations

$$
C_{2}(z)=\sum_{k=0}^{\infty}(-1)^{k} \frac{z^{2 k}}{(2 k !)^{2}}, \quad S_{2}(z)=\sum_{k=1}^{\infty}(-1)^{k-1} \frac{z^{2 k-1}}{((2 k-1) !)^{2}} .
$$

Proposition 2.1. The functions $E_{2}^{(k)}(z)$ defined in Proposition 1.2 possess the addition formulae

$$
E_{2}^{(k)}(x+y)=\sum_{n=-\infty}^{\infty} E_{2}^{(n)}\left(\frac{x}{2}\right) E_{2}^{(k-n)}\left(\frac{y}{2}\right) .
$$

The proof follows from (1.8). Indeed,

$$
\begin{aligned}
e^{2 t} e^{(x+y) / t} & =\sum_{-\infty}^{\infty} t^{n} E_{2}^{(n)}(2(x+y))=e^{t} e^{x / t} \cdot e^{t} e^{y / t} \\
& =\sum_{-\infty}^{\infty} t^{n} E_{2}^{(n)}(x) \sum_{-\infty}^{\infty} t^{m} E_{2}^{(m)}(y) .
\end{aligned}
$$

Let $F(x)=E_{2}(-x)$. It is easily seen that $F(x)$ has an infinite number of positive zeros, $\beta_{k}, k=1,2,3, \ldots$.

Proposition 2.2. If $\beta_{k}(k=1,2, \ldots)$ are the zeros of $F(x)$ then the functions $F\left(\beta_{k} x\right)$ satisfy the orthogonality relations

$$
\begin{aligned}
\int_{0}^{1} F\left(\beta_{n} x\right) F\left(\beta_{m} x\right) d x & =0, \quad n \neq m, \\
& =\left(F^{\prime}\left(\beta_{n}\right)\right)^{2}, \quad m=n .
\end{aligned}
$$

Proof. Let $F_{k}(x)=F\left(\beta_{k} x\right)$. Then $\left(x F_{k}^{\prime}(x)\right)^{\prime}=-\beta_{k} F_{k}(x)$ and the rest follows from the Sturm-Liouville Theory.

Proposition 2.3. With $F\left(\beta_{k} x\right)$ as in (2.5), we have the orthogonality relations

$$
\begin{aligned}
-\int_{0}^{1} \int_{0}^{1} \frac{F\left(\beta_{n} x y\right) F\left(\beta_{m} x y\right)}{\log (x y)} d x d y & =0, \quad n \neq m, \\
& =\left(F^{\prime}\left(\beta_{n}\right)\right)^{2}, \quad m=n .
\end{aligned}
$$

The proof follows by setting $x y=s, x=t$ and if necessary interchanging the order of integration.

REMARK. Computer studies by the author suggest that $E_{n}(-x), n \geqslant 2$, have an infinite number of positive zeros, $x_{k}$, with the asymptotic distribution $x_{k}=O\left(k^{n}\right)$. Also, curiously, whereas $E_{2}(-x)=O\left(x^{-1 / 4}\right)$ for large positive $x, E_{3}(-x)=O\left(x^{k}\right), k$ approximately equal to one, and $E_{4}(-x)=O\left(x^{k}\right)$ with $k$ greater than two.

3. Applications to second order p. d. equations. The differential equation

$$
L u=D u-v(x, y) u=h(x, y)
$$

where $D=\partial^{2} / \partial x \partial y$ is of importance as many partial differential equations of the second order may be transformed to this form by change of variables. Integrating both sides of (3.1) we obtain

$$
u(x, y)=H(x, y)+f(x)+g(y)+\int_{0}^{x} \int_{0}^{y} v(s, t) u(s, t) d s d t,
$$


where $H(x, y)=\int_{0}^{x} \int_{0}^{y} h(s, t) d s d t, f(x)$ and $g(y)$ arbitrary continuously differentiable functions of their arguments. This equation is of the form (1.10) and its solution is given by (1.11). Setting $H(x, y)=0$ in (3.2) we get the general solution of the homogeneous equation $L u=0$. Choosing $f(x)=g(y)=0$ we obtain a particular solution of the nonhomogeneous equation $L u=h(x, y)$. Denoting this solution by $u_{p}(x, y)$ we have

Proposition 3.1. The particular solution $u_{p}(x, y)$ of (3.1) satisfying the conditions $u_{p}(0, y)=u_{p}(x, 0)=0$ is given by

$$
\begin{aligned}
u_{p}(x, y) & =H(x, y)+\int_{0}^{x} \int_{0}^{y} v(s, t) E_{2}\left(\int_{s}^{x} \int_{t}^{y} v(p, q) d p d q\right) H(s, t) d s d t \\
& =\int_{0}^{x} \int_{0}^{y} E_{2}^{*}\left(\int_{s}^{x} \int_{t}^{y} v(p, q) d p d q\right) h(s, t) d s d t .
\end{aligned}
$$

The proof is immediate. The first of these is a particular case of (1.11) and the second follows from the first by an interchange in the order of integration assuming that this is possible.

REMARK. The second part of (3.3) is, mutatis mutandis, the usual form of the particular integral of the ordinary linear first-order differential equation and could have been obtained in exactly the same way. We have, however, chosen the method of recursion because it is simpler, more general, more direct and leads to a more general result. Needless to say, the r.h.s. of (3.1) may depend on $u(x, y)$.

The solution of the homogeneous equation $L u=0$ is easily obtained from (3.2) and (1.11).

As an example we consider the ultra-hyperbolic equation in four independent variables

$$
u_{x_{1} x_{1}}+u_{x_{2} x_{2}}-u_{y_{1} y_{1}}-u_{y_{2} y_{2}}+k u=f
$$

where $k$ and $f$ are functions of $x_{1}^{2}+x_{2}^{2}$ and $y_{1}^{2}+y_{2}^{2}$. The substitutions $x=x_{1}^{2}+x_{2}^{2}$, $y=y_{1}^{2}+y_{2}^{2}, x=(p+q)^{2}, y=(p-q)^{2}, u=w /\left(p^{2}-q^{2}\right)^{1 / 2}$ reduce (3.4) to

$$
w_{p q}+K w=F
$$

where $w=w(p, q), F=\left(p^{2}-q^{2}\right)^{1 / 2} f\left(p^{2}+q^{2}, p^{2}-q^{2}\right)$ and

$$
K=k\left(p^{2}+q^{2}, p^{2}-q^{2}\right)-p q /\left(p^{2}-q^{2}\right)^{2} .
$$

It is interesting to note that if $f=0$ then for a suitable choice of $k, K=0$ and the equation reduces to $w_{p q}=0$ which has a solution similar to d'Alembert's solution of the wave equation, namely

$$
u=\left(g\left(x^{1 / 2}+y^{1 / 2}\right)+h\left(x^{1 / 2}-y^{1 / 2}\right)\right) /(x y)^{1 / 2}
$$

where $x=x_{1}^{2}+x_{2}^{2}, y=y_{1}^{2}+y_{2}^{2}$ and $g, h$ arbitrary continuously differentiable functions of their arguments.

The general solution of (3.5) is easily obtainable and we shall not write it down here.

4. Equations of higher order. In this section we shall consider the linear equation

$$
L u=D^{n} u+a_{1} D^{n-1} u+\cdots+a_{n} u=f
$$


where $u=u(x, y), f=f(x, y), D=\partial^{2} / \partial x \partial y$ and $a_{1}, \ldots, a_{n}$ are constants. We shall obtain the general solution of this equation as the sum of the general solution of the corresponding homogeneous equation and a particular solution of the nonhomogeneous equation in much the same way as this is done for the corresponding ordinary differential equation.

DEFinition 2. A function $C=C(x, y)$ will be said to be a " $D$-constant" function if

$$
D\left(C E_{2}(\beta x y)\right)=\beta C E_{2}(\beta x y), \quad \beta=\text { constant } .
$$

The form of a $D$-constant function is readily found. From (1.11) we have

$$
C E_{2}(\beta x y)=g(x)+h(y)+\beta \int_{0}^{x} \int_{0}^{y}(g(s)+h(t)) E_{2}(\beta(x-s)(y-t)) d s d t,
$$

where $g(x)$ and $h(y)$ are arbitrary continuously differentiable functions.

Proposition 4.1. Let $\beta_{1}, \ldots, \beta_{k}(k \leqslant n)$ be the distinct roots of $P_{n}(\beta)=\beta^{n}+$ $a_{1} \beta^{n-1}+\cdots+a_{n}=0$ and let $m_{i}$ denote the multiplicity of the root $\beta_{i}$. Then the general solution of the homogeneous equation $\mathrm{Lu}=0$ is given by

$$
u(x, y)=\sum_{i=1}^{k} \sum_{j=1}^{m_{i}} u_{i}^{(j)}(x, y)
$$

where

$$
u_{i}^{(j)}(x, y)=\frac{\partial^{j-1}}{\partial \beta^{j-1}}\left[C_{i}^{(j)} E_{2}(\beta x y)\right]_{\beta=\beta_{i}},
$$

$i=1, \ldots, k, j=1, \ldots, m_{i}$ and $C_{i}^{(j)}$ are $D$-constant functions.

The proof is identical to that of the corresponding theorem for ordinary differential equations, [1, Chapter VI].

To obtain a particular solution of the nonhomogeneous equation (4.1) we shall adapt the method of variation of parameters. We shall seek a particular solution of the form

$$
u_{p}=\sum_{i=1}^{n} K_{i} u_{i}
$$

where $K_{i}=K_{i}(x, y)$ are functions to be determined and $u_{i}=E_{2}\left(\beta_{i} x y\right)$. We shall assume, to begin with, that all the roots of $P_{n}(\beta)$ are simple. The equations from which the $K_{i}$ are to be determined may be written may be written as

$$
\begin{gathered}
\sum_{i=1}^{n} D\left(K_{i} u_{i}\right)-\sum_{i=1}^{n} K_{i} D u_{i}=0 \\
\sum_{i=1}^{n} D\left(K_{i} D U_{i}\right)-\sum_{i=1}^{n} K_{i} D^{2} u_{i}=0 \\
\vdots \\
\sum_{i=1}^{n} D\left(K_{i} D^{n-1} u_{i}\right)-\sum_{i=1}^{n} K_{i} D^{n} u_{i}=f
\end{gathered}
$$


Using the fact that $D u_{i}=\beta_{i} u_{i}$ and solving for the quantities $D\left(K_{i} u_{i}\right)-\beta_{i} K_{i} u_{i}$ we get

$$
D\left(K_{i} u_{i}\right)-\beta_{i} K_{i} u_{i}=\frac{V_{i}}{V} f .
$$

where $V=V\left(\beta_{1}, \ldots, \beta_{n}\right)$ is the Vandermonde determinant and $V_{i}$ is the minor of the element in the $n$th row and $i$ th column. These equations are of the form (3.1) and solving these for the $K_{i} u_{i}$, using the first of (3.3) and the fact that $V_{1}+\cdots+V_{n}=0$, we obtain

$$
u_{p}=\int_{0}^{x} \int_{0}^{y} F(s, t) K(x, y ; s, t) d s d t
$$

where

$$
K(x, y ; s, t)=\frac{1}{V} \sum_{i=1}^{n} \beta_{i} V_{i} E_{2}\left(\beta_{i}(x-s)(y-t)\right),
$$

and

$$
F(x, y)=\int_{0}^{x} \int_{0}^{y} f(s, t) d s d t .
$$

The result can be extended to the case that $P_{n}(\beta)$ has equal roots by noting that in this case the r.h.s. of (4.6) assumes the indeterminante form $0 / 0$ and l'Hôpital's Rule may be used to obtain the result. This is not difficult to do using the particular properties of the Vandermonde determinant but for the sake of simplicity we shall not state the result here. In the case that $P_{n}(\beta)$ has complex roots, the addition formula (2.4) may be used to write the result in terms of real functions.

As an example the equation $D^{2} u+k^{2} u=f, k=$ constant, has the particular integral

$$
u_{p}=\int_{0}^{x} \int_{0}^{y} F(s, t) C_{2}(k(x-s)(y-t)) d s d t
$$

where $C_{2}$ is the function defined in (2.1).

REMARK. It is possible to extend the method of variation of parmeters to the case that (4.1) has variable coefficients but then the equations (4.4) are not so easily solved. We may also allow $D$ to be the general differential operator defined in $\S 1$.

\section{REFERENCES}

1. E. L. Ince, Ordinary differential equations, Dover, New York, 1956.

2. R. Courant and D. Hilbert, Methods of mathematical physics. vol. II, Interscience, New York, 1962.

3. A. N. Tikhonov and A. A. Samarski, Equations of mathematical physics, Macmillan, New York, 1963.

School of Mathematics, University of Minnesota, Minneapolis, Minnesota 55455

Current address: 6 Highview Drive, Doncaster, Victoria 3108, Australia 